\title{
Solar Orbiter's first Venus flyby: Observations from the Radio and Plasma Wave instrument
}

\author{
L. Z. Hadid ${ }^{1}$, N. J. T. Edberg ${ }^{2}$, T. Chust ${ }^{1}$, D. Píša ${ }^{3}$, A. P. Dimmock ${ }^{2}$, M. W. Morooka ${ }^{2}$, M. Maksimovic ${ }^{4}$, \\ Yu. V. Khotyaintsev ${ }^{2}$, J. Souček ${ }^{3}$, M. Kretzschmar ${ }^{5}$, A. Vecchio ${ }^{4,6}$, O. Le Contel ${ }^{1}$, A. Retino ${ }^{1}$, R. C. Allen ${ }^{7}$, \\ M. Volwerk ${ }^{8}$, C. M. Fowler ${ }^{9}$, L. Sorriso-Valvo ${ }^{2,10}$, T. Karlsson ${ }^{11}$, O. Santolík ${ }^{3,12}$, I. Kolmašová ${ }^{3,12}$, F. Sahraoui ${ }^{1}$, \\ K. Stergiopoulou ${ }^{2}$, X. Moussas ${ }^{13}$, K. Issautier ${ }^{4}$, R. M. Dewey ${ }^{14}$, M. Klein Wolt ${ }^{6}$, O. E. Malandraki ${ }^{15}$, E. P. Kontar ${ }^{16}$, \\ G. G. Howes ${ }^{17}$, S. D. Bale ${ }^{9,18}$, T. S. Horbury ${ }^{19}$, M. Martinović ${ }^{20}$, A. Vaivads ${ }^{2,21}$, V. Krasnoselskikh ${ }^{5}$, E. Lorfèvre ${ }^{22}$, \\ D. Plettemeier ${ }^{23}$, M. Steller ${ }^{8}$, Š. Štverák ${ }^{24,25}$, P. Trávníček ${ }^{9,25}$, H. O’Brien ${ }^{19}$, V. Evans ${ }^{19}$, V. Angelini ${ }^{19}$, \\ M. C. Velli ${ }^{26}$, and I. Zouganelis ${ }^{27}$
}

(Affiliations can be found after the references)

Received 30 March 2021 / Accepted 3 June 2021

\begin{abstract}
Context. On December 27, 2020, Solar Orbiter completed its first gravity assist manoeuvre of Venus (VGAM1). While this flyby was performed to provide the spacecraft with sufficient velocity to get closer to the Sun and observe its poles from progressively higher inclinations, the Radio and Plasma Wave (RPW) consortium, along with other operational in situ instruments, had the opportunity to perform high cadence measurements and study the plasma properties in the induced magnetosphere of Venus.

Aims. In this paper, we review the main observations of the RPW instrument during VGAM1. They include the identification of a number of magnetospheric plasma wave modes, measurements of the electron number densities computed using the quasi-thermal noise spectroscopy technique and inferred from the probe-to-spacecraft potential, the observation of dust impact signatures, kinetic solitary structures, and localized structures at the bow shock, in addition to the validation of the wave normal analysis on-board from the Low Frequency Receiver.

Methods. We used the data products provided by the different subsystems of RPW to study Venus' induced magnetosphere.

Results. The results include the observations of various electromagnetic and electrostatic wave modes in the induced magnetosphere of Venus: strong emissions of $\sim 100 \mathrm{~Hz}$ whistler waves are observed in addition to electrostatic ion acoustic waves, solitary structures and Langmuir waves in the magnetosheath of Venus. Moreover, based on the different levels of the wave amplitudes and the large-scale variations of the electron number densities, we could identify different regions and boundary layers at Venus.

Conclusions. The RPW instrument provided unprecedented AC magnetic and electric field measurements in Venus' induced magnetosphere for continuous frequency ranges and with high time resolution. These data allow for the conclusive identification of various plasma waves at higher frequencies than previously observed and a detailed investigation regarding the structure of the induced magnetosphere of Venus. Furthermore, noting that prior studies were mainly focused on the magnetosheath region and could only reach 10-12 Venus radii $\left(R_{V}\right)$ down the tail, the particular orbit geometry of Solar Orbiter's VGAM1, allowed the first investigation of the nature of the plasma waves continuously from the bow shock to the magnetosheath, extending to $\sim 70 R_{V}$ in the far distant tail region.
\end{abstract}

Key words. plasmas - waves - polarization

\section{Introduction}

The ESA/NASA Solar Orbiter spacecraft (Müller et al. 2020), the first mission to directly study the Sun's north and south poles up close and their connection to the inner heliosphere, was launched on February 10, 2020. In order to tighten the probe's orbit around the Sun and to gradually lift it out of the ecliptic plane, Solar Orbiter uses seven gravity-assists from Venus and one from Earth. The first Venus gravity assist manoeuvre (VGAM1) on December 27, 2020 provided the first opportunity for the field and particle instruments to operate within a planetary magnetosphere prior to the beginning of their nominal phase in December 2021. More specifically, the Radio and Plasma Wave (RPW) instrument (Maksimovic et al. 2020) was able to make its first continuous and high cadence measurements inside the magnetosphere of Venus. Noting that the instrument was designed to operate in the interplanetary medium, the VGAM1 allowed us to test the capabilities of RPW in conditions that are different than the solar wind. In addition, it allowed us (1) to cross-calibrate and test the operations of the different receivers, (2) to validate the wave normal analysis of the instrument in an environment where whistler mode emissions with well-known characteristics are present, and (3) to investigate the presence of dust impacts and lightning signatures on Venus near periapsis. Furthermore, this first Venus flyby provided a comprehensive set of plasma and wave data in different regions of Venus' induced magnetosphere from far downtail to the subsolar magnetosheath.

Since the 1960s, Venus has been investigated by various flyby missions (Mariner 2, 5 and 10, Galileo, and Cassini), landers (Venera 11-14), atmospheric probes, and orbiter missions (Pioneer Venus, Venus Express, and Akatsuki), out of these, Mariner 10 had the most similar flyby trajectory to Solar Orbiter and could study magnetic field fluctuations far downtail (Lepping \& Behannon 1978). Nevertheless, the nature of the high frequency plasma waves at Venus has been mainly studied by the Pioneer Venus Orbiter (PVO), as it was the only orbiter that carried a plasma wave instrument, the Orbiter Electric Field Detector (OEFD; Scarf et al. 1980b). Various types 
of propagation modes have been identified in different regions around the planet: $30 \mathrm{kHz}$ Langmuir oscillations were present in the foreshock, $5.4 \mathrm{kHz}$ Doppler-shifted electrostatic ion-acoustic waves were observed in the bow shock and appeared to be a prominent feature of the magnetotail boundary, and electromagnetic $100 \mathrm{~Hz}$ whistler waves were thought to be generated at the bow shock, propagating through the magnetosheath and eventually absorbed within the ionosphere (Scarf et al. 1979, 1980a; Strangeway 1991). However, the identification of these waves was not well determined as they were also suspected to be lower hybrid waves (Szegö et al. 1991), or ion acoustic waves (Strangeway \& Crawford 1993; Huba 1993). This ambiguity was a direct consequence of the absence of a wave magnetic field sensor on-board PVO and the limited frequency coverage of the OEFD instrument, that had only a 4 channel electric field receiver (E field wave power at $100 \mathrm{~Hz}, 730 \mathrm{~Hz}, 5.4 \mathrm{kHz}$, $30 \mathrm{kHz}$ ). Furthermore, after more than forty years long search for lightning on Venus, results obtained by the different spacecraft missions are still inconclusive. The most compelling indications of some kind of atmospheric electrical activity at Venus appear to be the Venera landers' detections of electromagnetic pulses at frequencies from 10 to $80 \mathrm{kHz}$ (Ksanfomaliti 1980). Their origin is questionable (Lorenz 2018); they might be assigned to interferences similarly as electric pulses found by Galileo (Gurnett et al. 1991). No lightning related signals were found during the Cassini Venus flyby in spite of the fact that the same instrument detected up to 70 sferics per second during the Earth flyby in 1999 (Gurnett et al. 2001). The whistler-mode waves at frequencies near $100 \mathrm{~Hz}$ recorded by Pioneer Venus and Venus Express were attributed to lightening (Russell et al. 2008). Nevertheless, a variety of other explanations were offered for these electromagnetic observations (Lorenz 2018). Up to now, not a single image from any spacecraft has been able to confirm the existence of lightning including an extensive a 5-years long search for lightning by the Akatsuki Venus orbiter (Takahashi et al. 2018). However, the modeling did not exclude electrical breakdown in the Venusian atmosphere and transient luminous events were forecasted to occur on Venus (Riousset et al. 2020).

Recently launched spacecraft missions that use Venus for gravity assist manoeuvres, provide a rare opportunity to study the electromagnetic environment of the planet. In particular, the FIELDS experiment (Bale et al. 2016) on-board Parker Solar Probe (PSP; Fox et al. 2016) provided the first simultaneous AC magnetic and electric field measurements at Venus, and revealed a strong kinetic-scale turbulence in the magnetosheath (Bowen et al. 2021) and the presence of kinetic-scale electric field structures such as plasma double layers near the bow shock (Malaspina et al. 2020; Goodrich et al. 2021). Moreover, BepiColombo on its way to Mercury, completed its first Venus flyby on October 15, 2020 and allowed to investigate the structure and the magnetic field properties of the induced magnetosphere of Venus (Volwerk et al. 2021a). Solar Orbiter with its VGAM1 and the high quality and broad frequency range of the RPW instrument allowed, to identify conclusively various propagation wave modes in different regions in the induced magnetosphere of Venus. Moreover, it offered a unique opportunity for investigating in situ the magnetosphere of Venus continuously from the bow shock down to the distant tail region, as far as 70 Venus Radii $\left(R_{V}, 1 R_{V}=6051 \mathrm{~km}\right)$ downstream.

Solar Orbiter entered the Venus' induced magnetosphere on the nightside southern hemisphere and travelled toward the northern hemisphere on December 27, 2020. It had its closest approach over the northern pole at an altitude of $13488 \mathrm{~km}$
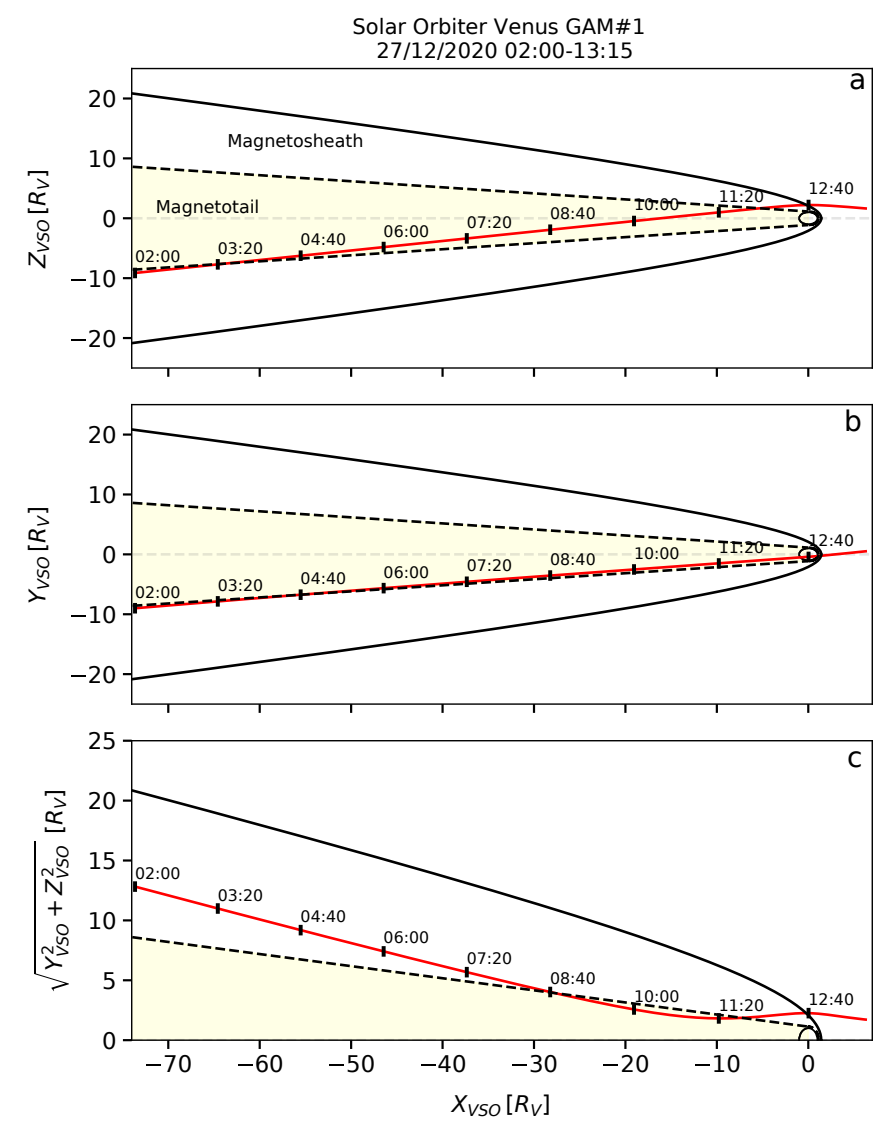

Fig. 1. Solar Orbiter trajectories (in red) projected into the VSO (a) $X Z$, (b) $X Y$ planes and (c) in cylindrical coordinates. The bow shock (black solid line) and the upper mantle boundary, also referred as the induced magnetospheric boundary (black dashed line) from Martinecz et al. (2009) are displayed.

$\left(\sim 2 R_{V}\right)$ near 12:39:20UT, crossed the bow shock outbound around 12:40 UT and continued upstream in the solar wind. The trajectory of the spacecraft in the Venus Solar Orbital (VSO) coordinates is shown in Fig. 1. The VSO system is analogous to the geocentric solar ecliptic coordinates where the $X_{\mathrm{VSO}}$ is directed from the center of the planet toward the Sun, $Z_{\mathrm{VSO}}$ is normal to the Venus orbital plane and positive toward the north celestial pole, and $Y_{\mathrm{VSO}}$ is positive in the direction opposite to orbital motion. The bow shock and upper mantle boundary (also known as the induced magnetosphere boundary) models (Martinecz et al. 2009) are included to indicate the approximate locations of Solar Orbiter encounters with these boundaries.

Our primary objective in this paper is to review the main observations of Solar Orbiter's RPW instrument during VGAM1. After a short description of the instrument in Sect. 2, we present, in Sect. 3, RPW observations: we show an overview of the electric and magnetic field frequency-time spectrograms (Sect. 3.1), the estimation of the total electron density (Sect. 3.2), and examples of electromagnetic waves (Sect. 3.3), various electrostatic waves (Sect. 3.4) and dust impact features (Sect. 3.5). Lastly, in Sect. 4 we conclude with the main RPW observations during the VGAM1.

\section{Instrumentation}

The RPW instrument is designed to measure the electric and magnetic fields of radio emissions and plasma waves across a 
broad range of frequencies, from quasi-DC to $16 \mathrm{MHz}$ for electric fields and from $1 \mathrm{~Hz}$ to $1 \mathrm{MHz}$ for magnetic fields. Details on RPW can be found in the extensive instrument description by Maksimovic et al. (2020). Briefly, the instrument includes a set of three monopole electric antennas (ANT) and a set of triaxial magnetic search coil units (Jannet et al. 2021) that are connected to a BIAS unit that monitors the potential of the electric antennas and a sub system of plasma and radio wave receivers: the thermal noise and high frequency receiver (TNR-HFR), the time domain sampler (TDS), and the low frequency receiver (LFR). TNR produces continuously quasi-instantaneous spectra for the electrostatic thermal noise and the magnetic field in the range of $4 \mathrm{kHz}-1 \mathrm{MHz}$, while HFR is a sweeping receiver, covering the frequency range from $500 \mathrm{kHz}$ to $16 \mathrm{MHz}$. TNR-HFR can measure Langmuir waves, solar radio bursts and the quasi-thermal noise from which the electron number density can be determined accurately (Sect. 3.2). The TDS enables high duty cycle waveform snapshots observation in the frequency range of $200 \mathrm{~Hz}-$ $200 \mathrm{kHz}$, resolving in particular plasma waves near the electron plasma frequency and voltage spikes associated with dust impacts. The LFR is a complex sub system designed to digitize and process signals from the electric antennas and the search coil magnetometer over a frequency range of quasi DC to $10 \mathrm{kHz}$. It is in particular used for wave normal analysis for characterizing the wave propagation and polarization properties (Sect. 3.3). It basically provides three different data products: waveforms, spectral matrices and basic wave parameters at different time and frequency resolutions. In order to cover continuously the entire frequency range from $\sim \mathrm{DC}$ up to $10 \mathrm{kHz}$, four data streams are provided simultaneously at different sampling frequencies: $F_{3}=16 \mathrm{~Hz}, F_{2}=256 \mathrm{~Hz}, F_{1}=4096 \mathrm{~Hz}$, and $F_{0}=24576 \mathrm{~Hz}$. A detailed and comprehensive description of LFR measurements capabilities and data processing can be found in Chust et al. (2021), Maksimovic et al. (2020).

Throughout the day of VGAM1, the RPW instrument suite operated in BURST mode. The LFR recorded continuous waveforms (CWFs) sampled at $F_{2}$, compressed spectral matrices (BP2) every $5 \mathrm{~s}$, and synthetic wave parameter sets (BP1) every $1 \mathrm{~s}$. These basic wave parameters, BP2 and BP1, were both calculated regularly on board from the F1 and F0 data streams, completing the frequency range up to $10 \mathrm{kHz}$. TDS was operating in a higher cadence mode with a regular waveform snapshot taken every $10 \mathrm{~s}$ at the rate of $262 \mathrm{ksps}$ and the length of $32 \mathrm{~ms}$ between 06:00 and 18:00 UT. TDS sampled two dipole antennas (V1-V3 and V2-V1) and one monopole antenna (V2). Beside regular snapshots, there were captured 343 on-board classified triggered snapshots with a length of $62 \mathrm{~ms}$. More detailed description of the TDS operation and in-flight performance is in Souček et al. (2021). TNR was configured to perform measurements by alternating different sensors in time on the two measurement channels 1 and 2. The HFR sensor operated only on V1-V2 sampled on channel 1. The power spectral densities measured by both TNR and HFR are obtained as an average over 16 measurements. This, together with the used configuration, resulted in an average time between two TNR spectra measured at the same sensor as follows: $4 \mathrm{~s}$ for V1-V2, $8 \mathrm{~s}$ for $\mathrm{V} 1$ and V3, and $2.7 \mathrm{~s}$ for the search coil. To keep the sampling time of a single HFR spectrum (always obtained as the average over 16 measurements) low, only 22 frequency bins where chosen. This resulted in a sampling time of $0.44 \mathrm{~s}$. However, since HFR measurements where alternated with TNR ones, the average time between two HFR spectra is larger and equal to $8 \mathrm{~s}$ (Vecchio et al. 2021; Maksimovic et al. 2021).

\section{Observations}

\subsection{RPW wave spectra}

The RPW observations during the first Venus flyby are summarized in Fig. 2. In order to have an overview of the various radio and plasma waves at the different frequency ranges and to check the consistency of the measurements between the different receivers, we resample at $4 \mathrm{~s}$ and combine the power spectral densities (PSDs) measured independently by LFR, TDS and TNR. Figure 2-a shows the combined electric field frequencytime spectrogram covering the frequency range from $8 \mathrm{~Hz}$ to $\sim 1 \mathrm{MHz}$ : LFR frequency range is from $6 \mathrm{~Hz}$ to $\sim 10 \mathrm{kHz}$, where the different data streams sampled at F2, F1 and F0 cover $[6 \mathrm{~Hz}-$ $102 \mathrm{~Hz}],[88 \mathrm{~Hz}-1752 \mathrm{~Hz}]$ and $[1584 \mathrm{~Hz}-10032 \mathrm{~Hz}]$ with frequency resolution of $4 \mathrm{~Hz}, 64 \mathrm{~Hz}$ and $384 \mathrm{~Hz}$, respectively. TDS covers frequencies from $\sim 9.9 \mathrm{kHz}$ up to $\sim 16 \mathrm{kHz}$ and TNR from $\sim 16.7 \mathrm{kHz}$ to $978 \mathrm{kHz}$. We note that HFR data are not included here as they required additional calibration and processing at the time of writing the paper. Figure 2-b shows the combined magnetic field spectral density for the same sampling time and frequency range as in panel a. LFR data are from $8 \mathrm{~Hz}$ to $\sim 9.8 \mathrm{kHz}$ and TNR data from $\sim 9.9 \mathrm{kHz}$ to $978 \mathrm{kHz}$. As one can note, for both spectrograms, the transition from one frequency range to another does not occur in a smooth manner, this is actually due to the fact that LFR and TNR are not sensing the same magnetic channels (Maksimovic et al. 2020). Nevertheless, qualitatively, clear signatures in the PSDs are consistently and continuously observed from the lower frequencies, as detected by LFR, up to the higher frequencies as detected by TDS and TNR, hence confirming the validity of these measurements. This is particularly apparent in the electric field data, where the bow shock crossing around 12:40 UT is clearly detected by all the receivers in addition to other narrowband and wideband wave signatures observed continuously from about $8 \mathrm{~Hz}$ up to $\sim 10 \mathrm{kHz}$. In both figures, the electron cyclotron $\left(f_{\mathrm{ce}}\right)$ and ion $\left(f_{\mathrm{pi}}\right)$ and electron plasma $\left(f_{\text {pe }}\right)$ frequencies which are useful in organizing a number of the plasma wave phenomena, are traced by black lines $\left(f_{\mathrm{ce}}<f_{\mathrm{pi}}<f_{\mathrm{pe}}\right)$. They are respectively given by $f_{\mathrm{ce}}=28 \mathrm{~B}$, where $\mathrm{B}$ is the magnetic field strength in $\mathrm{nT}$ measured by the magnetometer (MAG) instrument (Horbury et al. 2020), $f_{\mathrm{pe}}=$ $8980 n_{\mathrm{e}}$ and $f_{\mathrm{pi}}=214 n_{\mathrm{i}}\left(\right.$ assuming $\left.n_{\mathrm{i}}=n_{\mathrm{e}}\right)$, where $n_{\mathrm{e}}$ and $n_{\mathrm{i}}$ are the electron and ion number densities respectively, expressed in $\mathrm{cm}^{-3}$ (Sect. 3.2). We note that the above multiplicative constants, obtained combining various parameters, have physical dimension such as to obtain frequencies in $\mathrm{Hz}$.

The two spectrograms in Fig. 2 show the high frequency plasma waves observed in Venus' induced magnetosphere. Below the electron cyclotron frequency, various quasielectrostatic and electromagnetic modes can be observed. Moving from the nightside to the dayside, one can notice that in the distant magnetosheath region further tailward $\left(>50 R_{V}\right)$ the emissions are essentially electromagnetic of $\sim 30 \mathrm{~Hz}$, with some bursty electrostatic signatures. Deeper inside the magnetosheath and closer to Venys ( 09:00 UT-12:00 UT), there appears to be broadband electrostatic turbulence. A gradual increase in the electromagnetic signatures right inside the magnetosheath (between $\sim 12: 17$ UT and 12:36 UT) can be observed. In fact, both the bow shock (BS) and the magnetosheath (MS) are highlighted by abrupt and strong broadband wave activity in the electric and magnetic field spectra (as marked by the dashed white lines in Fig. 2). In Sect. 3.3 we discuss more in details these electromagnetic waves and show that they are predominantly circularly polarized whistler waves. These changes in the level and the amplitude of the wave activity could indicate the presence 


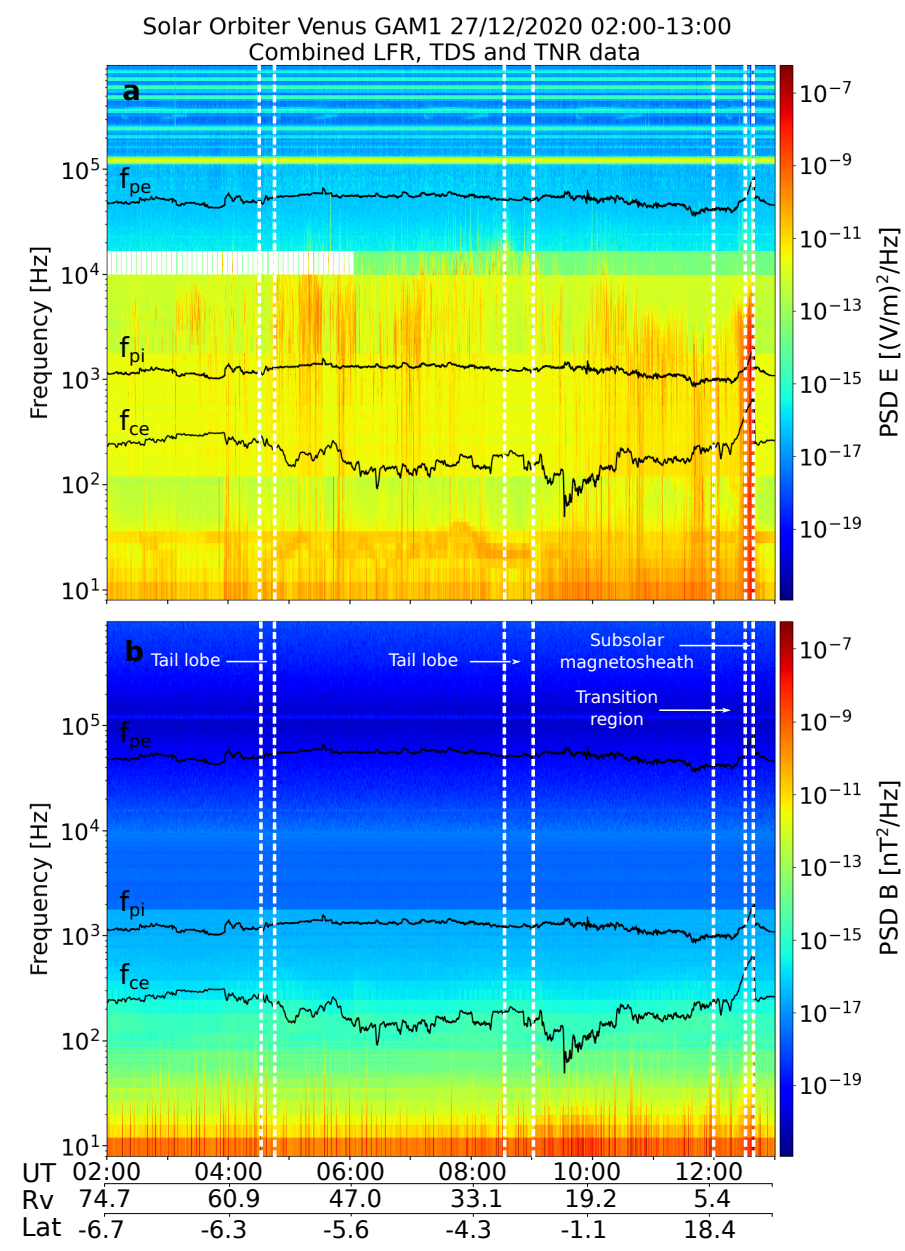

Fig. 2. Overview of the combined power spectral densities observed by Solar Orbiter's radio and plasma wave instrument during its first flyby at Venus. (a) The electric field spectrum, obtained by combining RPW subsystems data from LFR, TDS and TNR. (b) The magnetic field spectrum, obtained by combining RPW subsystems data from LFR and TNR. The electron cyclotron $\left(f_{\mathrm{ce}}\right)$ and ion $\left(f_{\mathrm{pi}}\right)$ and electron plasma $\left(f_{\mathrm{pe}}\right)$ frequencies are shown in black lines $\left(f_{\mathrm{ce}}<f_{\mathrm{pi}}<f_{\mathrm{pe}}\right)$. The vertical dashed lines delimit different regions in the induced magnetosphere of Venus.

of different regions in Venus' induced magnetosphere. This is discussed in more detail in Sect. 3.2. We note that the periodiclike variations around $20 \mathrm{~Hz}$ in the background of the electric field data are artificial noise. Their cause is still under investigation. Between $f_{\text {ce }}$ and $f_{\text {pe }}$ the waves become purely electrostatic with broadband emissions between $\sim 200 \mathrm{~Hz}$ and $\sim 10 \mathrm{kHz}$. These waves seem to be sporadic beyond $\sim 50 R_{V}$ in the distant tail region, then due to the Doppler-shift effect (not shwon here), gradually drift upward in frequency extending up to $\sim 30 \mathrm{kHz}$, notably between 07:00 UT and 09:00 UT. It is worth noting a clear decrease and absence of wave activity between 08:20 09:10 UT and 04:10-04:45 UT, in the electric field spectra from $\sim 8 \mathrm{~Hz}$ to $10 \mathrm{kHz}$ and the magnetic spectra from $\sim 8 \mathrm{~Hz}$ to $50 \mathrm{~Hz}$. This could be indicating crossings of the magnetotail boundary. In fact, the plasma waves at the tail boundary and in the magnetotail were investigated by Russell et al. (1981) who showed using PVO's OEFD data large fluctuations of electrostatic waves around $5 \mathrm{kHz}$ right outside the tail, with quieter levels within the tail itself. Langmuir waves could also be observed close to $f_{\mathrm{pe}}$ around $40 \mathrm{kHz}$ and $60 \mathrm{kHz}$ (Sect. 3.2). More discussion on the interpretation of some of the electromagnetic and electrostatic emissions is given below. At this stage of the analysis, no radio waves and lightening signatures could be observed above $f_{\mathrm{pe}}$, as the data need more calibration and processing. The horizontal lines above $120 \mathrm{kHz}$ are due to disturbances from the spacecraft and the periodic structures around $300 \mathrm{kHz}$ are due to the battery charging.

\subsection{Total electron density}

Using the quasi-thermal noise (QTN hereafter) spectroscopy technique, we could deduce the total electron density in the induced magnetosphere of Venus. The QTN technique has been used in a wide variety of space plasmas for in situ diagnostics (Meyer-Vernet \& Perche 1989; Meyer-Vernet et al. 1998, 2017; Moncuquet et al. 2005). The method is based on the analysis of the voltage power spectrum produced by the thermal motion of the charged particles of the plasma. These electrostatic fluctuations, which are completely determined by the particle velocity distributions, are detected by any sensitive radio receiver at the ports of a passive electric antenna. Therefore, the analysis of the QTN spectrum reveals in situ plasma properties such as the total electron density and bulk temperature (Meyer-Vernet \& Perche 1989; Meyer-Vernet et al. 2017). The method is relatively immune to the spacecraft perturbations since it senses a large plasma volume and can be used to crosscheck other sensors. For a weakly magnetized plasma where $f_{\text {ce }} \ll f_{\text {pe }}$, such as Venus, the QTN spectroscopy yields an accurate determination of the electron density since the electron thermal motions excite Langmuir waves, which produce a spectral peak just above the plasma frequency. Hence, the local electron density can be simply deduced from the tracking of the plasma line at $f_{\mathrm{pe}}\left(n_{\mathrm{e}} \propto f_{\mathrm{pe}}^{2}\right)$. In Fig. 3 we show the TNR time-frequency spectrogram during Solar Orbiter's VGAM1 on which the plasma line (highlighted in white) at $f_{\mathrm{pe}}$ emerges. Using a peak detection algorithm (Maksimovic et al. 2020, 2021), TNR-HFR subsystems are able to detect onboard the local plasma frequency from which $n_{\mathrm{e}}$ can be calculated. However, because of the very strong spurious spacecraft contamination around $120 \mathrm{kHz}$, the algorithm retrieved the plasma peak at $120 \mathrm{kHz}$ instead, and so the automatic detection was done on ground. In Fig. 4-a we show the electron number density during VGAM1 computed using the automatic (black) and manual (purple) detection of $f_{\mathrm{pe}}$ on ground. Even though the manual selection of $f_{\mathrm{pe}}$ results in a lower time resolution for $n_{\mathrm{e}}$, both measurements give consistent results hence confirming the accuracy and the robustness of the peak detection algorithm. The blue curve represents the electron density $n_{\mathrm{e}} \mathrm{S} / \mathrm{C}$ inferred from the spacecraft potential measurement (Khotyaintsev et al. 2021). As RPW was operating in BURST mode the spacecraft potential measurement could be provided at a sampling frequency of $256 \mathrm{~Hz}$. We note that the variation in the spacecraft potential gives direct estimates of the variation in $n_{\mathrm{e}}$ but not of its absolute value. After calibrating $n_{\mathrm{e} / \mathrm{C}}$ to $n_{\mathrm{e}} \mathrm{QTN}$, we get the electron number density at a high cadence of $256 \mathrm{~Hz}$ (Fig. 4).

The structure of the induced magnetosphere of Venus is quite evident in the dayside with a clear signature of the outbound bow shock around 12:40 UT. On average, the electron number density is $\sim 40 \mathrm{~cm}^{-3}$ inside the magnetosphere and decreases to $\sim 20 \mathrm{~cm}^{-3}$ in the solar wind. Moreover, one can see large scale variations in the density profile implying the presence of different regions and boundary layers. Figure 4-b, is a zoom on the time interval between 12:00 UT and 12:41 UT downstream of the bow shock. The magnetosheath region (12:37-12:40 UT) is characterized by a highly compressed and turbulent plasma 


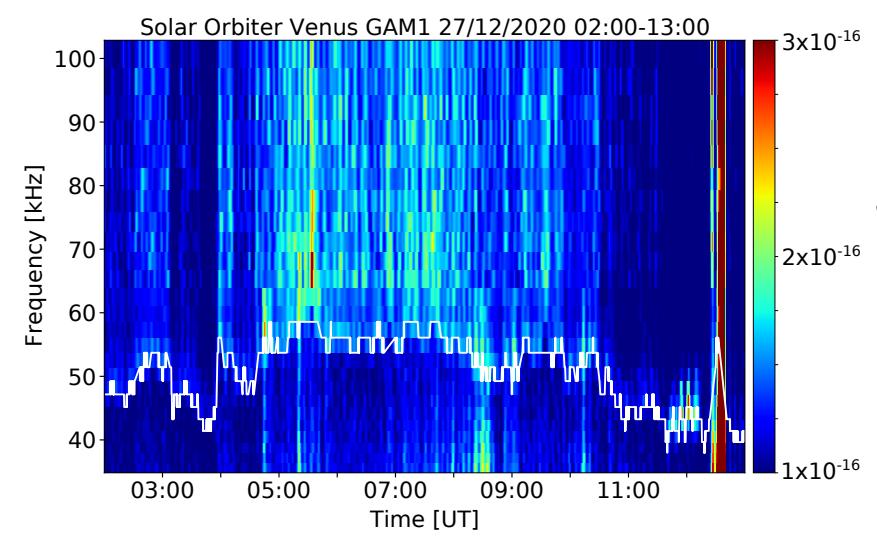

Fig. 3. TNR time-frequency spectrogram in which the plasma line at $f_{\mathrm{pe}}$ (highlighted in white) emerges between $40 \mathrm{kHz}$ and $60 \mathrm{kHz}$.

$\sim 80 \mathrm{~cm}^{-3}$, followed by a gradual decrease in $n_{\mathrm{e}}$ indicating the presence of a "transition region". This "transition region" was first observed by Venera 9 and 10 downstream of the bow shock (Gringauz et al. 1976; Verigin et al. 1978) and was shown to be characterized by a less dense, slower and hotter plasma than the magnetosheath. Unfortunately, the plasma temperature and velocity were not available during this flyby to support the identification of this region. Further toward the nightside, between 10:00 UT and 12:00 UT, as Solar Orbiter skimmed the upper mantle boundary (Fig. 1-c), $n_{\mathrm{e}}$ increases up to $\sim 40 \mathrm{~cm}^{-3}$ and stays relatively uniform in the distant magnetosheath region (05:00-10:00 UT). Nevertheless, a clear local decrease in $n_{\mathrm{e}}$, can be noticed $\sim 08: 20-09: 10$ UT and $\sim 04: 10-04: 45$ UT, highlighted in yellow in Fig. 4-a). As shown in Allen et al. (2021) and Volwerk et al. (2021b), this region coincides as well with an increase of the magnetic field strength, hence indicating the passage of Solar Orbiter into the lobe region of the magnetotail. Small-scale structures and sharp discontinuities can also be observed at the inbound leg of the orbit between 02:00 and 05:00 indicating the presence of a highly structured transition layer between the solar wind and the far distant tail region. These structures, observed as well with the magnetic field data, are discussed more in detail in Volwerk et al. (2021b). Nevertheless, these variations could be also due to the nonstationarity (or the flappening) of the tail region of Venus. The absence of the bulk plasma data during this flyby makes it hard to determine and identify the different boundary crossings during VGAM1.

In Fig. 5, we expand the time interval across the outbound bow shock crossing, where large and small scale structures occur at the foot and the ramp of the bow shock. The electron density and AC electric field (perpendicular and parallel components) are shown in panels a and $b$, respectively. Since only two components of the electric field are measured, $E_{\perp}$ and $E_{\|}$are defined as the components of $E$ along and perpendicular to the magnetic field in the $Y Z$ plane. The electron density exhibits large amplitude fluctuations across the shock, which have periods around $0.7 \mathrm{~s}$. These features are also seen in the MAG data and occur simultaneously to the electron density variations. These observations could be a piece of evidence of ion-inertial-scale waves known as "shock ripples" that have been also observed at the Earth quasi-perpendicular bow shock using MMS data (Gingell et al. 2017; Johlander et al. 2018) or large amplitude whistler structures (Wilson 2017), which can also be a signature of nonstationarity (Krasnoselskikh et al. 2002; Dimmock et al. 2019). Moreover, the electric field displays significant perturbations across the shock and clear wave activity in the frequency

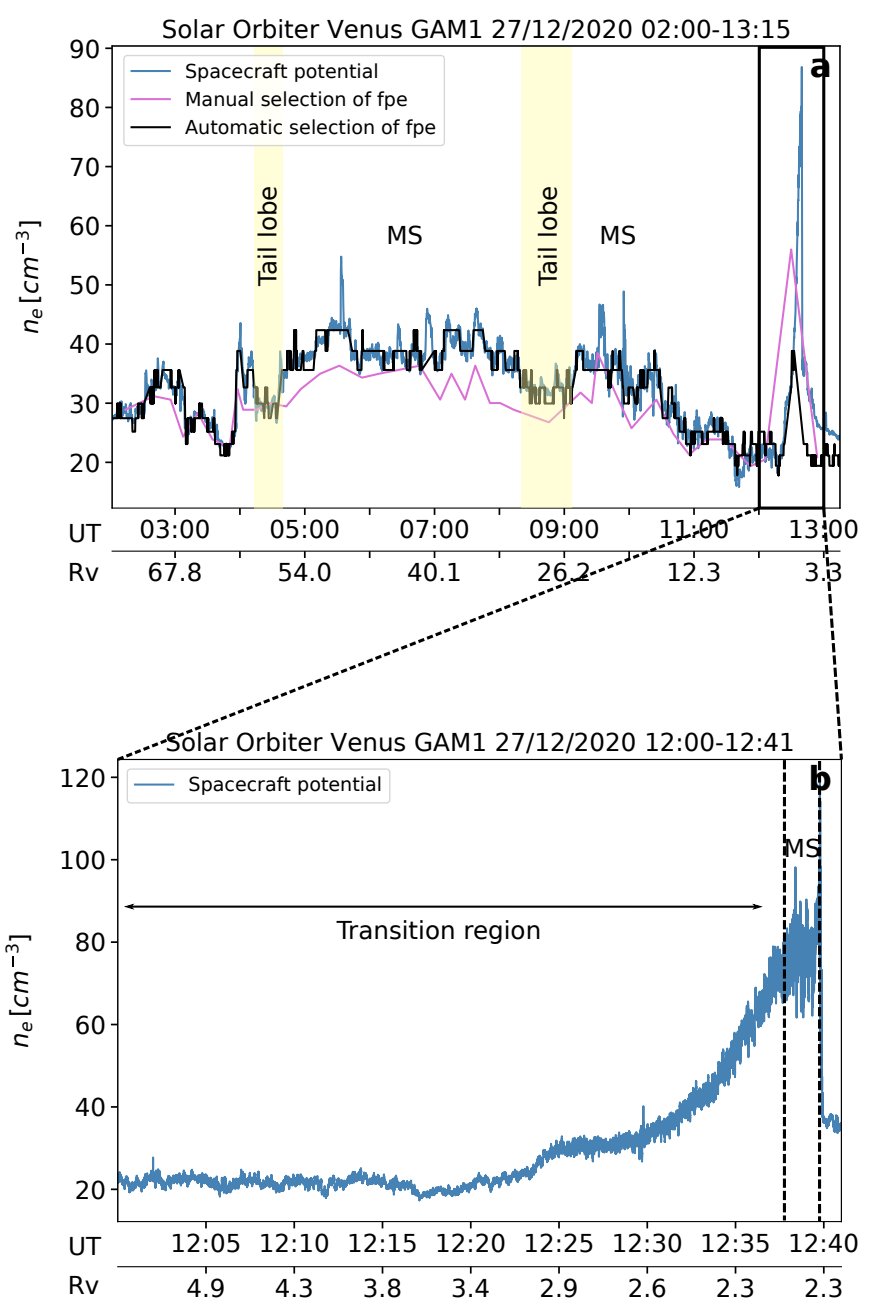

Fig. 4. (a) Electron density as a function of time using the quasi-thermal noise spectroscopy technique with an automatic (black) and the manual (magenta) detection of the plasma peak. The blue curve represents the electron number densities inferred using the spacecraft potential data. (b) Zoom on $n_{\mathrm{e}}$ between 12:00 and 12:41 UT. The dashed lines mark boundary crossings and transitions between different regions from the solar wind, to the magnetosheath (MS) and the lobe of the tail region (highlighted in yellow).

range $30-120 \mathrm{~Hz}$ (Sect. 3.3). A thorough analysis of these large amplitude features and the smaller-scale whistler waves across this interval is provided by Dimmock et al. (2021).

\subsection{Wave normal analysis: Electromagnetic whistler waves}

As mentioned in Sect. 2, the RPW/LFR subsystem is capable of observing and analyze a variety of low-frequency plasma waves up to $10 \mathrm{kHz}$, especially when they are electromagnetic, by determining their propagation characteristics, such as measuring one component of the Poynting vector and estimating the wave normal vector and the phase velocity. This has been demonstrated by Chust et al. (2021) with several examples of whistler mode waves selected after the near Earth commissioning phase of Solar Orbiter.

In Fig. 6, we show an example of LFR's wave normal analysis by selecting clear wave signatures that are present in the magnetosheath just inward of the bow shock. The top two panels show total power spectral density of the electric and magnetic field fluctuations (PE and PB respectively), with intensity 

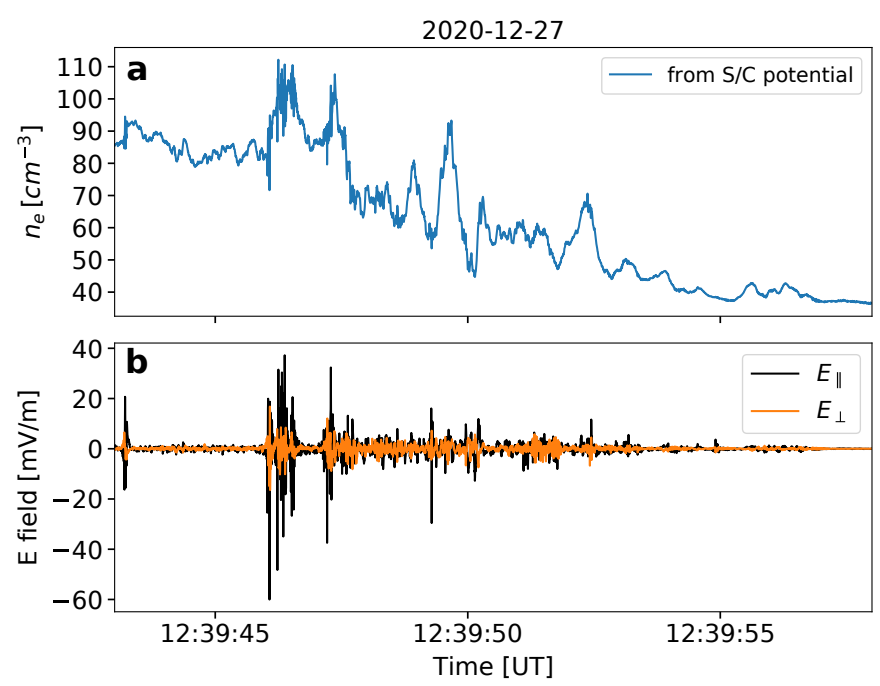

Fig. 5. (a) Electron density inferred from the spacecraft potential and (b) the AC electric field (perpendicular and parallel components).

indicated by the color bar to the right. The third panel shows the degree of polarization of the wave (DOP) calulated as a function of the trace of the magnetic spectral matrices (Samson 1973; Samson \& Olson 1980). This DOP parameter basically validates (or not) the assumption regarding the presence of a single plane wave, for a given frequency. A pure plane wave is shown by $\mathrm{DOP} \sim 1$ whereas a non pure or multiple waves is indicated by $\mathrm{DOP} \leq 0.6$. In order to highlight the most polarized waves, we only plot the data points that satisfy the condition DOP $\geq 0.9$. Using the Means method (Means 1972), the polarization properties of the waves are computed such as the ellipticity (ellip) and the angle between the wave normal vector and the local magnetic field $\left(\theta_{\mathbf{n} \cdot \mathbf{B}_{0}}\right)$. The wave normal vector $(\mathbf{n})$ is defined as the unit vector perpendicular to the plane of polarization of the wave and oriented in such a way that the rotation of the magnetic field fluctuation within that plane is right-handed $(\mathrm{RH})$, or counterclockwise, with respect to it. An estimate of a signed phase velocity $\left(v_{\varphi 1}\right)$, based on the Maxwell-Faraday equation and the measurement of two electric field components, is displayed in the last panel. Positive values indicate a wave propagation along $\mathbf{n}$, while negative values in the opposite direction. The different methods and formulas used to derive these parameters are described in detail by Chust et al. (2021). We note that these formulas are essentially relevant for the analysis of electromagnetic waves and are not able to account for electrostatic waves. Therefore, due to the presence of large electrostatic waves (second panel) superimposed on the electromagnetic waves (first panel), we had to slightly modify the formula (15) in Chust et al. (2021) to calculate $v_{\varphi 1}$, by not dividing the spectral cross-correlation terms by their corresponding correlation coefficients. This was intended to compensate for the digital artifacts that produce lower correlations. But here, these weak correlations between the electric and magnetic components should be taken as such, and the modulus of the spectral cross-correlation terms alone is a better estimate of the fraction of electric fluctuations that is correlated with the magnetic fluctuations. The modified expression that we use in this work is as follows:

$v_{\varphi 1}=\frac{n_{Y}\left\langle\hat{E}_{Z} \hat{B}_{X}^{*}\right\rangle_{t}-n_{Z}\left\langle\hat{E}_{Y} \hat{B}_{X}^{*}\right\rangle_{t}}{\left\langle\hat{B}_{X} \hat{B}_{X}^{*}\right\rangle_{t}}$

where $\hat{B}_{i}$ and $\hat{E}_{j}$ are the fast Fourier transform of the electric and magnetic field vector components respectively, $n_{i}$ is the wave

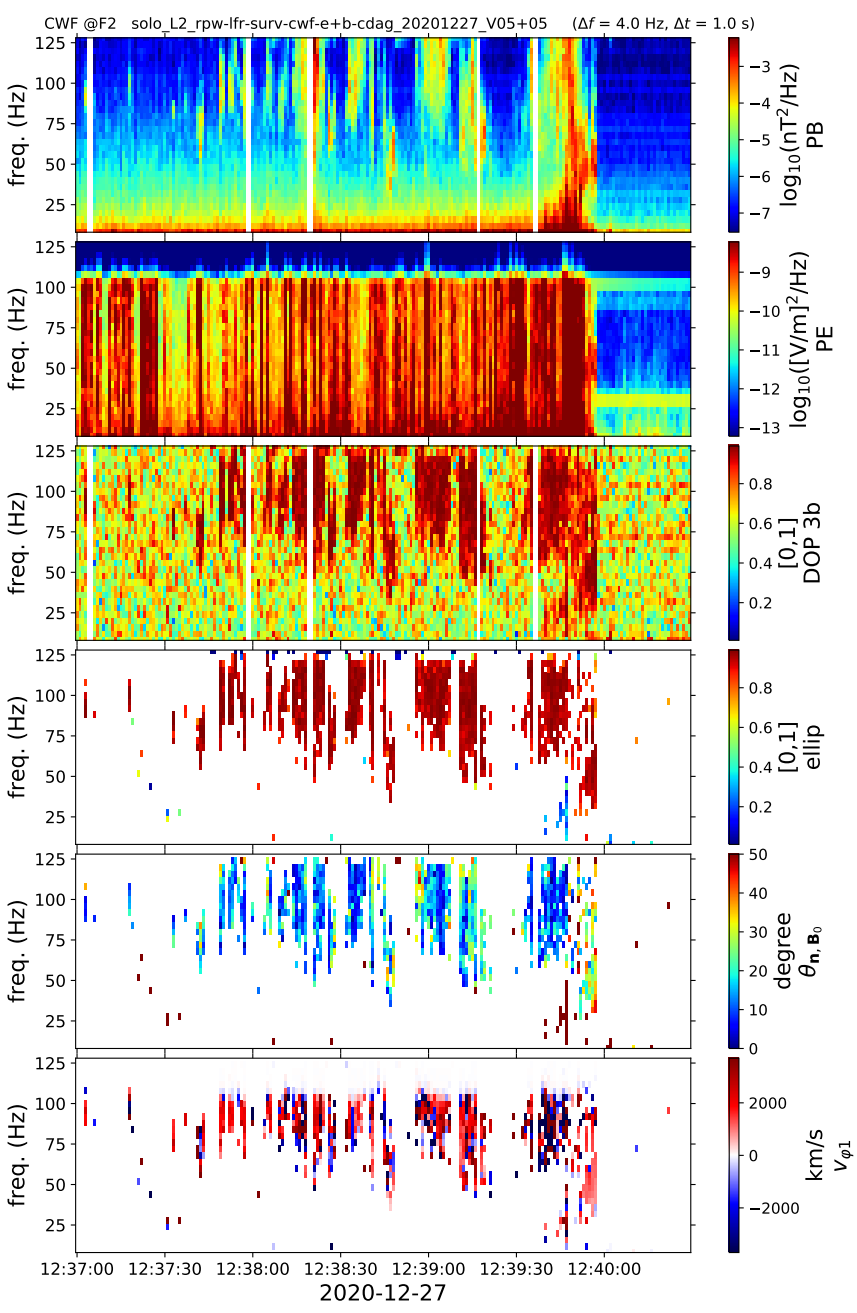

Fig. 6. Example of an electromagnatic whistler waves event observed in the magnetosheath of Venus. From top to bottom: the total power of the measured magnetic (PB) and electric (PE) fields fluctuations, the degree of polarization (DOP), the wave ellipticity (ellip), the angle between the wave normal vector $(\mathbf{n})$ and the background magnetic field vector $\mathbf{B}_{0}$ and the signed phase velocity $\left(v_{\varphi 1}\right)$.

normal vector of the component $i$, the subscript symbol ${ }^{*}$ denotes the complex conjugate and \langle\rangle$_{t}$ the average over time.

Very strong and bursty broadband emissions can be observed below the electron cyclotron and proton plasma frequencies in the PB at the bow shock and in the magnetosheath between 12:38-12:40 UT around $25 \mathrm{~Hz}$ and drift upward to $125 \mathrm{~Hz}$. Strong signatures can also be observed in the PE at all the frequency ranges. As the DOP is $\sim 1$ this confirms the presence of single plane waves that are electromagnetic in nature. Moreover, by looking at the other parameters we can say that the observed emissions are all circularly polarized (ellip $\approx 1$ ) and propagate with wave normal vectors nearly parallel to the background magnetic field $\left(\theta_{\mathbf{n} \cdot \mathbf{B}_{0}} \leq 30^{\circ}\right)$. The averaged positive sign of $v_{\varphi 1}$ (last panel) means that the wave vector is also inclined by the same angle $\theta_{\mathbf{n} \cdot \mathbf{B}_{0}}$ from the background magnetic field, which is in this case oriented approximately along the $-Z$ direction in the spacecraft reference frame (not shown). As $\theta_{\mathbf{n} \cdot \mathbf{B}_{0}}<90^{\circ}$, then $\mathbf{n}$ and $\mathbf{B}_{0}$ are pointing in the same direction, by definition this corresponds to a $\mathrm{RH}$ polarization of the wave magnetic field with respect to $\mathbf{B}_{0}$. We note that $\mathbf{B}_{0}$ is oriented along $-Z$ in the spacecraft reference frame (MAG observations not shown here). All these characteristics, with the fact that $f \approx 0.1 f_{\mathrm{ce}}$ 
hence indicate that the waves are most likely electromagnetic $\sim 100 \mathrm{~Hz}$ whistler mode waves propagating from the bow shock inward. To confirm this we compare the estimated phase speed of the waves (last panel) with the theoretical phase speed of the quasi-parallel whistler mode waves in a dense redplasma $\left(f_{\text {ce }} \ll f_{\text {pe }}\right)$ given by $V_{\mathrm{p}}=\frac{c \sqrt{f\left(f_{\mathrm{ce}}-f\right)}}{f_{\mathrm{pe}}}$ (Stix 1992) where $c$ is the speed of light. Assuming, $f=0.1 f_{\text {ce }}$, and using $f_{\text {ce }}=755.8$ $\mathrm{Hz}, f_{\mathrm{pe}}=80307.6 \mathrm{~Hz},\left\|\mathbf{B}_{0}\right\|=27 \mathrm{nT}$, and $n_{0}=80 \mathrm{~cm}^{-3}$, we find $V_{\mathrm{p}} \approx 893 \mathrm{~km} \mathrm{~s}^{-1}$ which is sensibly lower than the observed phase speed of the waves. This difference has probably several causes related to the electric field data: (1) The electric field data have been calibrated here with effective antenna lengths $L_{\text {eff }}=9.1 \mathrm{~m}$, while it is already clear from previous observations of whistler mode waves (Kretzschmar et al. 2021; Chust et al. 2021) that for $n_{0}=80 \mathrm{~cm}^{-3}$, they should be close to their physical limit of $14 \mathrm{~m}$. The electric field fluctuations are therefore probably overestimated by about $30-35 \%$. (2) Due to some asymmetry in the electric antenna configuration, it is also a fact that the measurement of the $Z$-component $\left(E_{Z}\right)$ of the electric field fluctuations is not measured as good as the $Y$-component $\left(E_{Y}\right)$ (Chust et al. 2021; Khotyaintsev et al. 2021; Steinvall et al. 2021). It is precisely for this reason that Chust et al. (2021) proposed an alternative estimate of the wave phase velocity $\left(v_{\varphi 2}\right)$, based on the $E_{Y}$ measurement only. Nevertheless, the computation of $v_{\varphi 2}$ in our case (not shown) does not seem to bring a significant improvement. (3) The remaining difference could then most likely come from the presence of strong electrostatic waves, as already mentioned above. One can think that it could also change the sign of $v_{\varphi 1}$, not only its magnitude. But in this case it should give erratic results with randomly distributed positive and negative signs, which is not really the case. There is a clear predominance of positive signs.

In conclusion, even if the magnitude of $v_{\varphi 1}$ has a large error, we believe that its sign on average is correct and that the waves correspond well to whistler mode waves. The possibility that these observations represent whistlers generated by hypothetical lightning discharges on Venus is, however, excluded, since the waves propagate toward the planet, not from it.

These emissions are probably generated at the bow shock and propagate downstream in the magnetosheath of Venus, as previously observed by Pioneer Venus OEFD instrument (Sect. 1). A detailed discussion regarding the generation mechanisms of these waves is given by Dimmock et al. (2021). This analysis could be extended in the future to the higher frequencies $(>100 \mathrm{~Hz})$ using LFR's BP2 and BP1 at F1, to investigate any possible coupling between the observed $\sim 100 \mathrm{~Hz}$ whistler waves and higher frequency electrostatic waves.

\subsection{Electrostatic broadband and narrow band emissions}

An interesting feature of the electric field spectrogram (Fig. 2-a), are the broadband emissions between $1 \mathrm{kHz}$ up to about $10 \mathrm{kHz}$ that are mainly observed in the magnetosheath and the tail region between 05:00 12:30. From inspection of the magnetic field data in Fig. 2-b, it is clear that these emissions are purely electrostatic. In order to investigate more in detail the nature of these emissions, we examine the underlying waveforms from the TDS high resolution snapshot measurements. In the following subsections we show and discuss three examples of different types of electrostatic waves observed by RPW/TDS: electron phase-space holes, ion acoustic waves and Langmuir waves.

\subsubsection{Solitary waves: Electron phase-space holes}

The electrostatic solitary waves (ESWs), originally labeled as "Broadband Electrostatic Noise", are thought to be associated with regions carrying field-aligned currents and electron and ion beams and were shown to be important in acceleration mechanisms, such as wave-particle interactions and magnetic reconnection (Cattell et al. 2005). They have been detected in various planetary environments, at Earth from several spacecraft (Geotail, Polar, Wind, FAST, Cluster, THEMIS, and MMS) in different boundary layers such as the plasma sheet in the geomagnetic tail (Gurnett et al. 1976), the auroral zone (Gurnett \& Frank 1977) and the turbulent magnetosheath (Matsumoto et al. 1997). They have also been detected at Saturn and near its icy moon Enceladus (Williams et al. 2006; Pickett et al. 2015), in the wake of Jupiter's moon Europa (Kurth et al. 2001), in the lunar wake of Earth's Moon (Hashimoto et al. 2010), at an interplanetary shock (Williams et al. 2006) and more recently near the bow shock of Venus using PSP data (Malaspina et al. 2020). These waves consist of bursty broadband features in the electric field spectra and are identified in the corresponding waveform data as "bipolar" pulses of two half-sinusoids of opposite signs that are isolated either in time or in amplitude from other wave activity (Matsumoto et al. 1994; Ergun et al. 1998; Franz et al. 1998). Many studies have interpreted these nonlinear electrostatic waves as electron phase-space holes (EHs) propagating parallel to the magnetic field, such as (Malaspina et al. 2014).

In Fig. 7, we show an example of ESWs detected by TDS receiver around 08:02 close to the upper mantle boundary (between the magnetosheath and the lobe region). One can clearly see five structures with peak-to-peak amplitude higher than a few $\mathrm{mV} \mathrm{m}^{-1}$ and a characteristic timescale $\sim 0.5 \mathrm{~ms}$. Their characteristic frequency $\left(f_{\mathrm{ESW}}\right)$ as seen by an observer at rest can be estimated as the inverse ratio of their size $\left(L_{\mathrm{ESW}}\right)$ over their speed $\left(V_{\mathrm{ESW}}\right)$. As discussed in Franz et al. (2000), the parallel scale size of electron phase space holes is $\sim 2 \lambda_{D}$, where the Debye length (in cm) $\lambda_{D}=11.95 \sqrt{T_{\mathrm{e}} / n_{\mathrm{e}}}$, and the speed of the electron phase space holes is a fraction of the electron thermal speed (in $\mathrm{cm} \mathrm{s}^{-1}$ ) $V_{\mathrm{e}}=$ $6.74 \times 10^{5} \sqrt{T_{\mathrm{e}}}$, where $T_{e}$ is the electron temperature in Kelvins, and $n_{\mathrm{e}}$ is the electron density in $\mathrm{cm}^{-3}$. Assuming $V_{\mathrm{ESW}}=0.1 V_{\mathrm{e}}$, we obtain $f_{\mathrm{ESW}} \sim 2.81 \times 10^{3} \sqrt{n_{\mathrm{e}}}$. Using $n_{\mathrm{e}}=35 \mathrm{~cm}^{-3}$ (Fig. 4-a at 10:03) we find $f_{\mathrm{ESW}}=1.66 \mathrm{kHz}$ or a characteristic time scale of $\sim 0.6 \mathrm{~ms}$. This is very consistent with the observed time scale of the ESWs in Fig. 7, hence implying that these structures could be identified as electron phase-space holes.

\subsubsection{Ion acoustic waves}

Enhanced Doppler-shifted ion-acoustic waves (IAWs) have been identified in the Venus tail region by the PVO electric field instrument and were associated with changing ion distributions (Intriligator \& Scarf 1982, 1984). The coincident signatures in the electric field and in the ion distributions have been suggested to be indicative of the tail boundary region (not shown here) and to have implications of wave-particle interactions. Such emissions are ubiquitous in the solar wind and widely observed by the RPW/TDS (Píša et al. 2021).

In Fig. 8 we show an example of multiple IAWs identified visually around 08:05 in the same region of the observed ESWs. IAWs are observed sporadically in discrete packets with amplitudes that rarely exceed $3 \mathrm{mV} \mathrm{m}^{-1}$. They are characterized by packet durations on the order of $5 \mathrm{~ms}$, which indicates that their generation mechanism may be short-lived and impulsive. Similar IAWs but with much higher amplitude up to $100 \mathrm{mV} / \mathrm{m}$ 


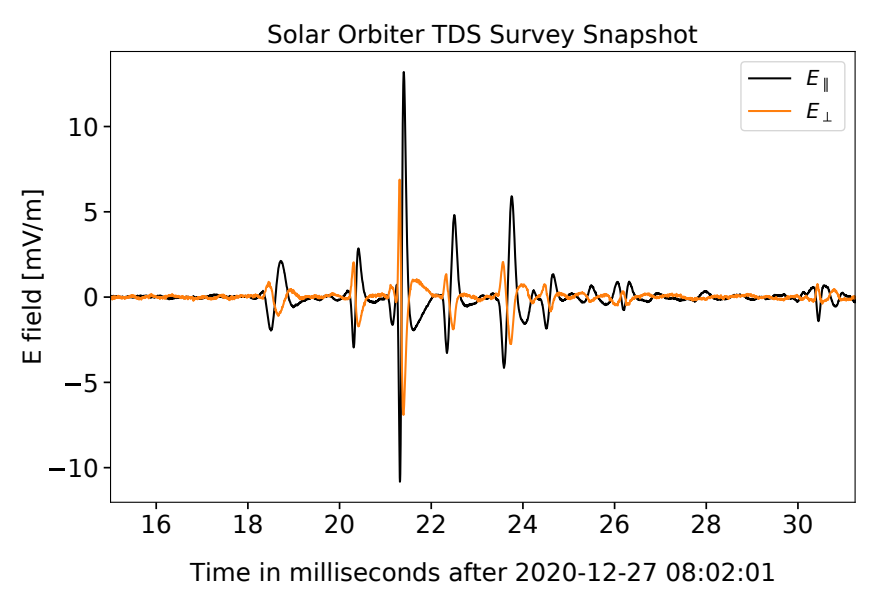

Fig. 7. Perpendicular (orange) and parallel (black) components of the electric TDS snapshot waveforms showing an example of an electrostatic solitary structures (electron phase-space holes).

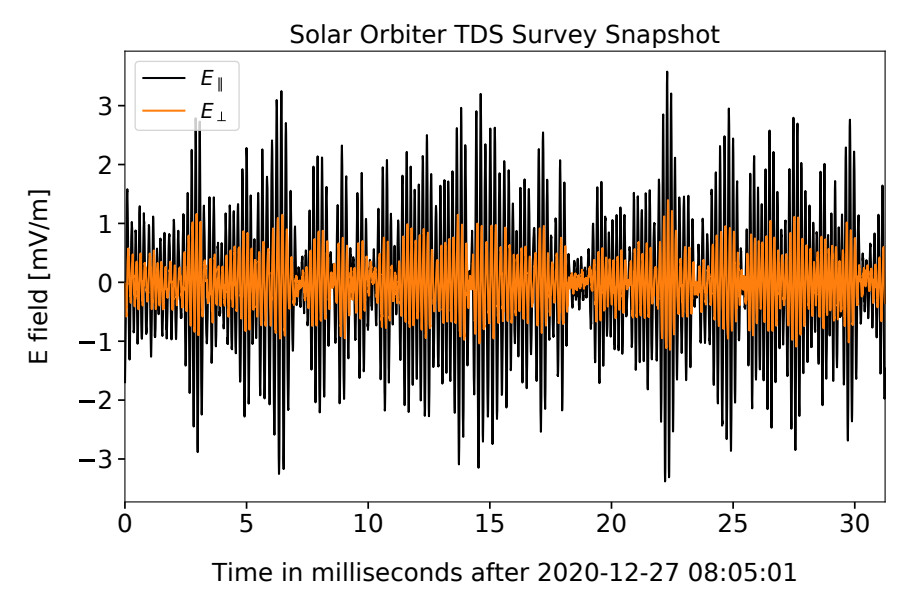

Fig. 8. Perpendicular (orange) and parallel (black) components of the electric field to the ambient magnetic field with intense ion-acoustic waves as recorded by TDS.

were detected by MMS in relation with a large scale KelvinHelmholtz instability at the Earth's magnetopause dusk flank (Wilder et al. 2016). By looking at the electric field power spectral density (Fig. 2), one can see the broadband power signature extending above the ion plasma frequency (black line), suggesting that these waves are Doppler-shifted IAWs. Such waves grow for a temperature ratio between ions and electrons greater than 1 (Kindel \& Kennel 1971) which in absence of particle measurement cannot be confirmed yet. Different plasma processes, including lower frequency plasma waves, that play a role in the acceleration of the supra-thermal ions have been investigated by Allen et al. (2021). The role of these high frequency waves in accelerating the particles (Szegö et al. 1991) during the VGAM1 have yet to be studied and will be the focus of future investigations.

\subsubsection{Langmuir waves}

Langmuir waves, also known as electron plasma oscillations, are electrostatic waves, that are generated around the electron plasma frequency by supra-thermal electron beams via the bump-on-tail instability (Briand et al. 2007). They have been extensively studied in the Earth magnetosphere, whereas around other planetary environments they are mostly used as a tool to
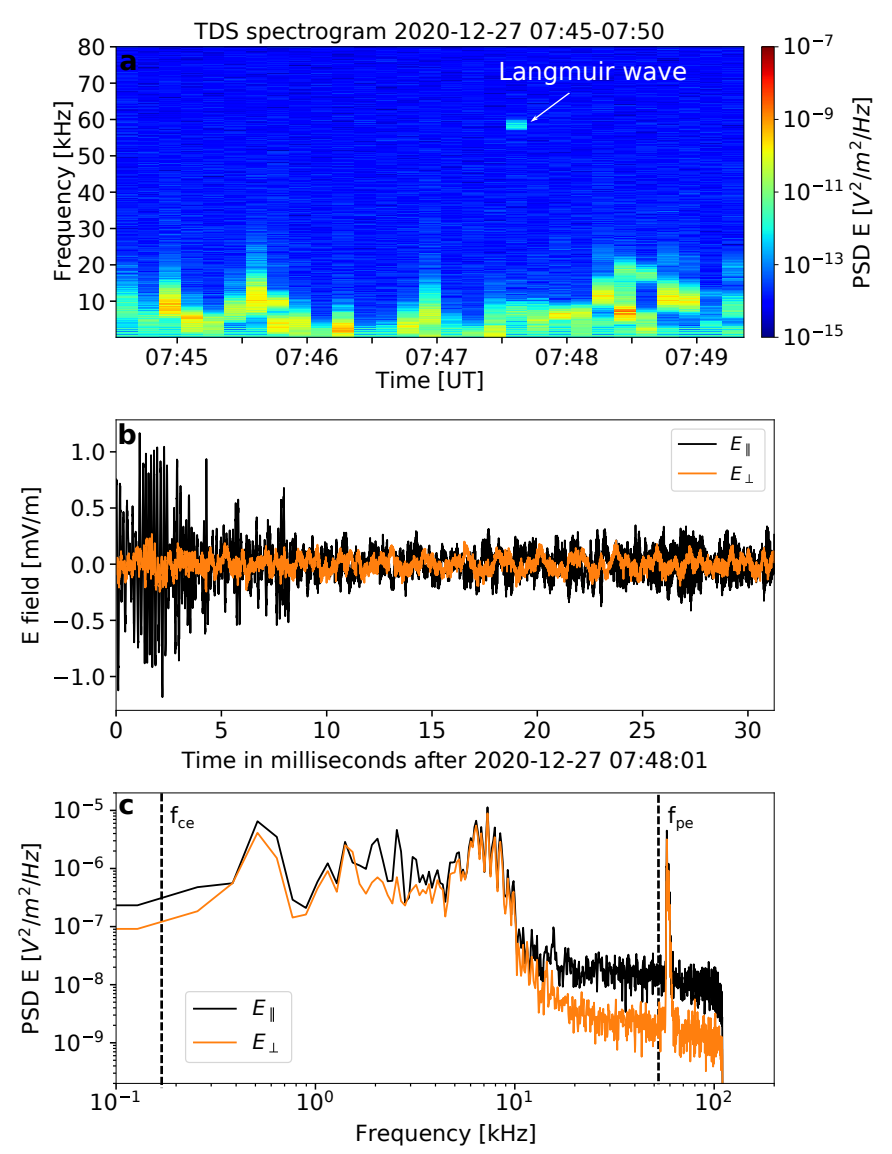

Fig. 9. (a) High-resolution frequency-time spectrogram of a Langmuir wave detected by the TDS receiver. (b) Langmuir emission electric field waveforms, perpendicular $\left(E_{\perp}\right)$ and parallel $E_{\|}$to the background magnetic field. (c) The power spectral densities of $E_{\perp}$ and $E_{\|}$. The vertical line $\sim 56 \mathrm{kHz}$ represents the electron plasma frequency $\left(f_{\mathrm{pe}}\right)$ detected by RPW/TNR.

diagnose the electron plasma density. A comprehensive review on the Langmuir waves across the heliosphere can be found in Briand (2015), Graham et al. (2021). Around Venus, using PVO data, Ho et al. (1993) reported the observation of Langmuir activity in the induced magnetotail around $\sim 30 \mathrm{kHz}$ and around $5.4 \mathrm{kHz}$. The propagation of these waves have also been observed upstream of the Venusian bow shock around $20 \mathrm{kHz}$, by the plasma wave experiments on-board Galileo and the Cassini spacecraft during their gravity assist manoeuvres at Venus and were thought to be produced by the solar wind energetic electrons streaming into the solar wind from the bow shock (Hospodarsky et al. 1994, 2006).

In Fig. 9 panel a, we show the frequency-time spectrogram of the TDS data. A clear narrow-band signature can be observed around 07:48:01 at $60 \mathrm{kHz}$ in the magnetosheath close to the lobe region (Fig. 4). In panel b, the perpendicular and parallel components to the background magnetic field, of the electric field waveform data are shown and the corresponding spectral densities are plotted in panel c. A distinct frequency component is evident between $58 \mathrm{kHz}$ and $60 \mathrm{kHz}$, just above the electron plasma frequency $\sim 56 \mathrm{kHz}$ as expected (marked by the vertical line). This suggests that the observed waves are Langmuir waves that could be produced by supra-thermal electrons. Furthermore, one can notice a broad feature below $10 \mathrm{kHz}$, which is due to the superposition of more intense low-frequency waves before $10 \mathrm{~ms}$ as shown in panel $b$. 


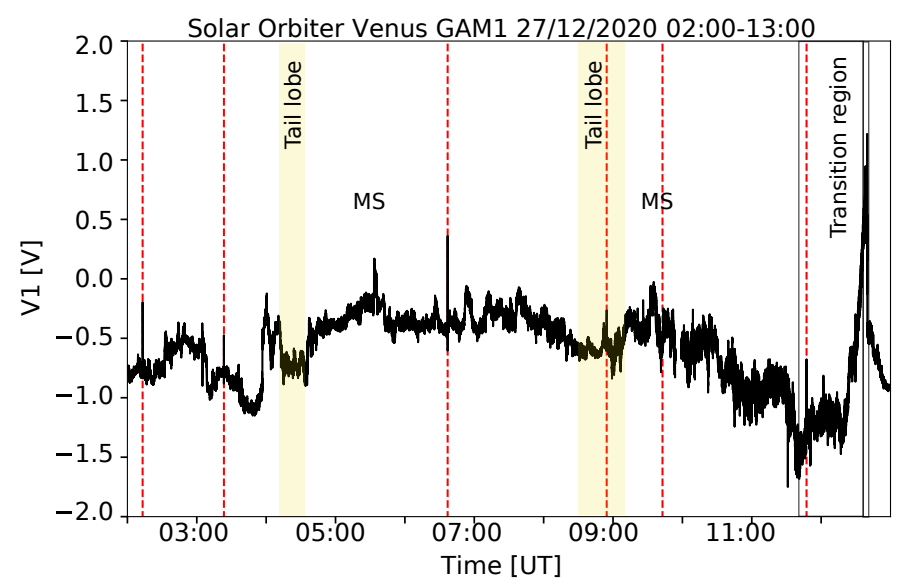

Fig. 10. Probe-to-spacecraft potential measured by the RPW antenna system and the BIAS unit. The red dashed lines denote the location of the dust impacts observed as distinct peaks. The color bars highlight the different regions in Venus' induced magnetosphere as shown in Fig. 4 the tail lobe (yellow), the magnetosheath (MS) and the transition region.
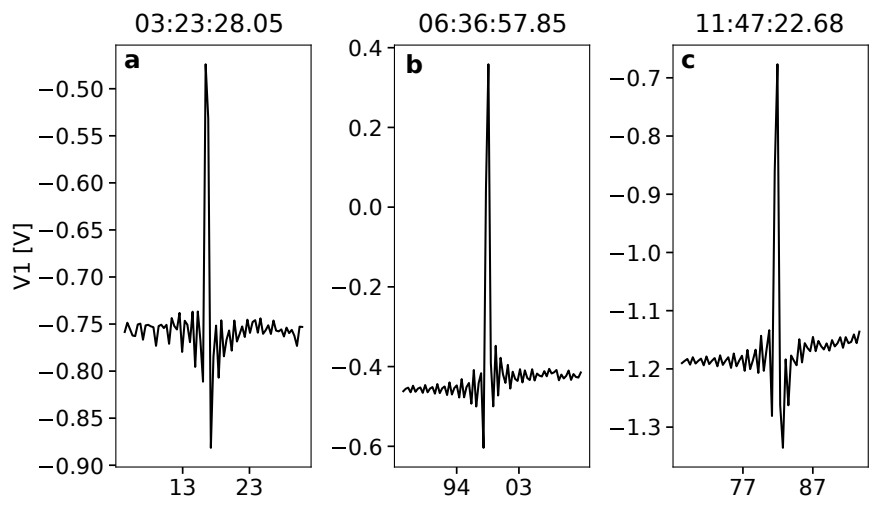

Time in milliseconds

Fig. 11. Examples of dust impact signatures expanded from Fig. 10 at (a) 03:23:28 UT, (b) 06:36:57 and (c) 11:47:22.

\subsection{Dust impacts}

During this first gravity assist manoeuvre of Venus, the Solar Orbiter RPW instrument also detected dust impacts inside the planet's induced magnetosphere (Fig. 10). In the absence of dedicated dust detectors, the electric antenna measurements provide the only opportunity to monitor dust particles in the space plasma environment. This method has been widely applied since the 1980s allowing to estimate the mass and size distribution of the impacting dust particles from the observed characteristics of the electric field signal (Gurnett et al. 1997; Meyer-Vernet 2001; Ye et al. 2014, 2018). One of the main mechanisms believed to convert the particle's kinetic energy of the dust into an electrical signal is the impact ionization process which generates clouds of electrons and ions around the spacecraft body (Gurnett et al. 1983; Aubier et al. 1983; Meyer-Vernet 1985). Because of the very large relative velocity between the spacecraft and the particles, when a dust particle hits the spacecraft, it instantly vaporizes, forming a hot ionized gas with free electrons and positive or negative ions that expand away from the impact site (Drapatz \& Michel 1974). These charged particles are hence attracted to, or repulsed from, the spacecraft surface depending on its electric potential relative to the surrounding ambient plasma. This induces an abrupt changes in the spacecraft potential which is then observed in the voltage data (e.g., escaping electrons generate a positive spacecraft signal). Recently, Mann et al. (2019) reviewed our current knowledge on dust antenna measurements, modeling works, and their prospect to inner heliospheric missions. Since TDS is designed to capture waveforms snapshots, it is well adapted to resolve voltage spikes associated with dust impacts. Thereby, Zaslavsky et al. (2021) provided a first analysis of dust measurements recorded by TDS, along Solar Orbiter's orbit, with promising results on the impact rate and dust grains radial velocities.

Figure 10 shows the continuous waveforms sampled at $F_{2}=$ $256 \mathrm{~Hz}$ of the Solar Orbiter's probe-to-spacecraft potential measured by the RPW antenna system and the BIAS unit. Impulsive signals with well-defined peaks can be clearly observed in different regions of Venus' induced magnetosphere (magnetosheath, tail lobe region and the far distant tail) reflecting the presence of dust impacts. Furthermore, the overall shape and structure of the different signatures are very similar (Fig. 11) as they are predominantly bipolar, characterized by a long peak of $\sim 10 \mathrm{msec}$ followed by a shorter one with opposite polarity lasting $\sim 2$ msec (Fig. 11-a-c), or the opposite, characterized by a first peak lasting $\sim 2 \mathrm{msec}$ then followed by a longer second peak of opposite polarity lasting at least several milliseconds (Fig. 11-b). These changes correspond to different phases of the impact process (impact cloud generation, electron escape, ion escape and spacecraft potential in equilibrium), and the shape of these signatures (positive peak, negative peak, or bipolar peaks) reflects the potential of the spacecraft (positive, negative, or zero) (Pantellini et al. 2012; Zaslavsky 2015). The origin and the estimation of the mass and the size distribution of the observed dust will be the subject of a separate study as more detailed analysis is required and careful distinction between dust and other wave features needs to be considered.

\section{Conclusions}

In this paper we presented an overview of a comprehensive set of the plasma wave observations obtained during the first Venus flyby of Solar Orbiter by the Radio and Plasma Wave instrument. The unique orbit geometry allowed us to investigate and characterize the induced magnetosphere of Venus for the first time as far as $70 R_{V}$. Furthermore, the high quality of the RPW instrument allowed us to identify decisively different structures and propagation modes at Venus, in particular over its north pole and all along the boundary of the tail region. We present the plasma density profile with large- and small-scale variations reflecting different boundary layer crossings. Moreover, we show examples of various types of waves and structures observed at the bow shock, downstream in the subsolar magnetosheath and close to the lobe region at different radial distances such as electromagnetic $\sim 100 \mathrm{~Hz}$ whistler waves, electrostatic $\sim 2 \mathrm{kHz}$ electron phase-space holes, $\sim 10 \mathrm{kHz}$ ion acoustic waves and $\sim 60 \mathrm{kHz}$ Langmuir waves. Additional investigations of the statistical occurrence of these waves and their role in the energy transport and dissipation will be done in future studies. Moreover, the next Venus flybys of Solar Orbiter will allow complementary analysis regarding the nature of the waves and the plasma properties in the induced magnetosphere of Venus.

Acknowledgements. Solar Orbiter is a mission of international cooperation between ESA and NASA, operated by ESA. The RPW instrument has been designed and funded by CNES, CNRS, the Paris Observatory, The Swedish National Space Agency, ESA-PRODEX and all the participating institutes. 
RCA is supported under NASA contract NNN06AA01C and thanks NASA headquarters and the NASA/GSFC Solar Orbiter project office for their continuing support. Solar Orbiter is a space mission of international collaboration between ESA and NASA, operated by ESA. This work is supported by the Swedish National Space Agency grant 20/136. Solar Orbiter magnetometer operations are funded by the UK Space Agency (grant ST/T001062/1). Tim Horbury is supported by STFC grant ST/S000364/1. APD was funded by the Swedish Civil Contingencies Agency (grant 2016-2102) and Swedish National Space Agency (Grant 2020-00111).

\section{References}

Allen, R. C., Mason, G. M., Ho, G. C., et al. 2021, A\&A, 656, L2 (SO Cruise Phase SI)

Aubier, M. G., Meyer-Vernet, N., \& Pedersen, B. M. 1983, Geophys. Res. Lett., 10,5

Bale, S. D., Goetz, K., Harvey, P. R., et al. 2016, Space Sci. Rev., 204, 49

Bowen, T. A., Bale, S. D., Bandyopadhyay, R., et al. 2021, Geophys. Res. Lett., 48, e2020GL090783

Briand, C. 2015, J. Plasma Phys., 81, 325810204

Briand, C., Mangeney, A., \& Califano, F. 2007, Phys. Lett. A, 368, 82

Cattell, C., Dombeck, J., Wygant, J., et al. 2005, J. Geophys. Res.: Space Phys., 110

Chust, T., Kretzschmar, M., Graham, D. B., et al. 2021, A\&A, 656, A17 (SO Cruise Phase SI)

Dimmock, A. P., Russell, C. T., Sagdeev, R. Z., et al. 2019, Sci. Adv., 5,

Dimmock, A. P., Khotyaintsev, Yu. V., Lalti, A., et al. 2021, A\&A, submitted (SO Cruise Phase SI)

Drapatz, S., \& Michel, K. W. 1974, Z. Naturforsch. A, 29, 870

Ergun, R. E., Carlson, C. W., McFadden, J. P., et al. 1998, Geophys. Res. Lett., 25,2041

Fox, N., Velli, M., Bale, S., et al. 2016, Space Sci Rev, 204, 7

Franz, J. R., Kintner, P. M., \& Pickett, J. S. 1998, Geophys. Res. Lett., 25, 1277

Franz, J. R., Kintner, P. M., Seyler, C. E., Pickett, J. S., \& Scudder, J. D. 2000 Geophys. Res. Lett., 27, 169

Gingell, I., Schwartz, S. J., Burgess, D., et al. 2017, J. Geophys. Res.: Space Phys., 122, 11,003

Goodrich, K. A., Bonnell, J. W., Curry, S., et al. 2021, Geophys. Res. Lett., 48, e2020GL090329

Graham, D. B., Khotyaintsev, Yu. V., Vaivads, A., et al. 2021, A\&A, 656, A23 (SO Cruise Phase SI)

Gringauz, K. I., Bezrukikh, V. V., Breus, T. K., et al. 1976, Plasma Observations Near Venus Onboard the Venera 9 and 10 Satellites by Means of Wide-Angle Plasma Detectors (American Geophysical Union (AGU)), 918

Gurnett, D. A., \& Frank, L. A. 1977, J. Geophys. Res. (1896-1977), 82, 1031

Gurnett, D. A., Frank, L. A., \& Lepping, R. P. 1976, J. Geophys. Res. (18961977), 81, 6059

Gurnett, D. A., Shawhan, S. D., \& Shaw, R. R. 1983, J. Geophys. Res.: Space Phys., 88, 329

Gurnett, D. A., Kurth, W. S., ROUX, A., et al. 1991, Science, 253, 1522

Gurnett, D. A., Ansher, J. A., Kurth, W. S., \& Granroth, L. J. 1997, Geophys Res. Lett., 24, 3125

Gurnett, D. A., Zarka, P., Manning, R., et al. 2001, Nature, 409, 313

Hashimoto, K., Hashitani, M., Kasahara, Y., et al. 2010, Geophys. Res. Lett. 37

Ho, C. M., Strangeway, R. J., \& Russell, C. T. 1993, Geophys. Res. Lett., 20, 2775

Horbury, T. S., OBrien, H., Carrasco Blazquez, I., et al. 2020, A\&A, 642, A9

Hospodarsky, G. B., Gurnett, D. A., Kurth, W. S., et al. 1994, J. Geophys. Res.: Space Phys., 99, 13363

Hospodarsky, G. B., Kurth, W. S., Gurnett, D. A., et al. 2006, Planetary Radio Emissions VI, Proceedings of the 6th International Workshop held at Graz, 67

Huba, J. D. 1993, Geophys. Res. Lett., 20, 1751

Intriligator, D. S., \& Scarf, F. L. 1982, Geophys. Res. Lett., 9, 1325

Intriligator, D. S., \& Scarf, F. L. 1984, J. Geophys. Res.: Space Phys., 89, 47

Jannet, G., Dudok de Wit, T., Krasnoselskikh, V., et al. 2021, J. Geophys. Res.: Space Phys., 126, e2020JA028543

Johlander, A., Vaivads, A., Khotyaintsev, Y. V., et al. 2018, Plasma Phys. Controlled Fusion, 60, 125006

Khotyaintsev, Yu. V., Graham, D. B., Vaivads, A., et al. 2021, A\&A, 656, A19 (SO Cruise Phase SI)

Kindel, J. M., \& Kennel, C. F. 1971, J. Geophys. Res. (1896-1977), 76, 3055

Krasnoselskikh, V. V., Lembège, B., Savoini, P., \& Lobzin, V. V. 2002, Phys. Plasmas, 9, 1192
Kretzschmar, M., Chust, T., Krasnoselskikh, V., et al. 2021, A\&A, 656, A24 (SO Cruise Phase SI)

Ksanfomaliti, L. V. 1980, Nature, 284, 244

Kurth, W., Gurnett, D., Persoon, A., et al. 2001, Planet. Space Sci., 49, 345 Magnetospheres of the Outer Planets (Part I)

Lepping, R. P., \& Behannon, K. W. 1978, J. Geophys. Res.: Space Phys., 83, 3709

Lorenz, R. D. 2018, Prog. Earth Planet. Sci., 5

Maksimovic, M., Bale, S. D., Chust, T., et al. 2020, A\&A, 642, A12

Maksimovic, M., Souček, J., Chust, T., et al. 2021, A\&A, 656, A41 (SO Cruise Phase SI)

Malaspina, D. M., Andersson, L., Ergun, R. E., et al. 2014, Geophys. Res. Lett., 41, 5693

Malaspina, D. M., Goodrich, K., Livi, R., et al. 2020, Geophys. Res. Lett., 47, e2020GL090115

Mann, I., Nouzák, L., Vaverka, J., et al. 2019, Ann. Geophys., 37, 1121

Martinecz, C., Boesswetter, A., Fränz, M., et al. 2009, J. Geophys. Res. Planets, 114, E00B30

Matsumoto, H., Kojima, H., Miyatake, T., et al. 1994, Geophys. Res. Lett., 21, 2915

Matsumoto, H., Kojima, H., Kasaba, Y., et al. 1997, Adv. Space Res., 20, 683 Results of the IASTP Program

Means, J. D. 1972, J. Geophys. Res. (1896-1977), 77, 5551

Meyer-Vernet, N. 1985, Adv. Space Res., 5, 37

Meyer-Vernet, N. 2001, Spacecraft Charging Technology, Proceedings of the Seventh International Conference held 23-27 April, 2001 at ESTEC, Noordwijk, the Netherlands, 635

Meyer-Vernet, N., \& Perche, C. 1989, J. Geophys. Res.: Space Phys., 94, 2405

Meyer-Vernet, N., Hoang, S., Issautier, K., et al. 1998, Measuring Plasma Parameters With Thermal Noise Spectroscopy (American Geophysical Union (AGU)), 205

Meyer-Vernet, N., Issautier, K., \& Moncuquet, M. 2017, J. Geophys. Res.: Space Phys., 122, 7925

Moncuquet, M., Lecacheux, A., Meyer-Vernet, N., Cecconi, B., \& Kurth, W. S. 2005, Geophys. Res. Lett., 32, L20S02

Müller, D., St. Cyr, O. C., Zouganelis, I., et al. 2020, A\&A, 642, A1

Pantellini, F., Belheouane, S., Meyer-Vernet, N., \& Zaslavsky, A. 2012, Astrophys. Space Sci., 341, 309

Pickett, J. S., Kurth, W. S., Gurnett, D. A., et al. 2015, J. Geophys. Res.: Space Phys., 120, 6569

Píša, D., Souček, J., Santolík, O., et al. 2021, A\&A, 656, A14 (SO Cruise Phase SI)

Riousset, J. A., Nag, A., \& Palotai, C. 2020, Icarus, 338, 113506

Russell, C. T., Luhmann, J. G., Elphic, R. C., \& Scarf, F. L. 1981, Geophys. Res. Lett., 8, 843

Russell, C. T., Zhang, T. L., \& Wei, H. Y. 2008, J. Geophys. Res. Planets, 113, E00B05

Samson, J. C. 1973, Geophys. J. R. Astron. Soc., 34, 403

Samson, J. C., \& Olson, J. V. 1980, Geophys. J. Int., 61, 115

Scarf, F. L., Taylor, W. W. L., \& Green, I. M. 1979, Science, 203, 748

Scarf, F. L., Taylor, W. W. L., \& Virobik, P. F. 1980a, IEEE Trans. Geosci. Remote Sens., GE-18, 36

Scarf, F. L., Taylor, W. W. L., Russell, C. T., \& Elphic, R. C. 1980b, J. Geophys. Res.: Space Phys., 85, 7599

Souček, J., Píša, D., Kolmasova, I., et al. 2021, A\&A, 656, A26 (SO Cruise Phase SI)

Steinvall, K., Khotyaintsev, Yu V., Cozzani, G., et al. 2021, A\&A, 656, A9 (SO Cruise Phase SI)

Stix, T. 1992, Waves in Plasmas (American Inst. of Physics)

Strangeway, R. J. 1991, Space Sci. Rev., 55, 275

Strangeway, R. J., \& Crawford, G. K. 1993, Geophys. Res. Lett., 20, 1211

Szegö, K., Shapiro, V. S., Shevchenko, V. I., et al. 1991, Geophys. Res. Lett., 18 2305

Takahashi, Y., Sato, M., Imai, M., et al. 2018, Earth. Planets Space, 70, 88

Vecchio, A., Maksimovic, M., Krupar, V., et al. 2021, A\&A, 656, A33 (SO Cruise Phase SI)

Verigin, M. I., Gringauz, K. I., Gombosi, T., et al. 1978, J. Geophys. Res.: Space Phys., 83, 3721

Volwerk, M., Mautner, D., Wedlund, C. S., et al. 2021a, Ann. Geophys., 39, 239

Volwerk, M., Horbury, T. S., Woodham, L. D., et al. 2021b, A\&A, 656, A11 (SO Cruise Phase SI)

Wilder, F. D., Ergun, R. E., Schwartz, S. J., et al. 2016, Geophys. Res. Lett., 43, 8859

Williams, J. D., Chen, L.-J., Kurth, W. S., Gurnett, D. A., \& Dougherty, M. K. 2006, Geophys. Res. Lett., 33, L06103

Wilson III, L. B., Koval, A., Szabo, A., et al. 2017, J. Geophys. Res. (Space Phys.), 122, 9115 
Ye, S.-Y., Gurnett, D. A., Kurth, W. S., et al. 2014, J. Geophys. Res.: Space Phys., 119,6294

Ye, S.-Y., Kurth, W. S., Hospodarsky, G. B., et al. 2018, J. Geophys. Res.: Space Phys., 123, 4952

Zaslavsky, A. 2015, J. Geophys. Res.: Space Phys., 120, 855

Zaslavsky, A., Mann, I., Souček, J., et al. 2021, A\&A, 656, A30 (SO Cruise Phase SI)

${ }^{1}$ LPP, CNRS, Observatoire de Paris, PSL Research University, Sorbonne Université, École Polytechnique, Institut Polytechnique de Paris, 91120 Palaiseau, France e-mail: lina.hadid@lpp.polytechnique.fr

2 Swedish Institute of Space Physics, Box 537, 75121 Uppsala Sweden

${ }^{3}$ Department of Space Physics, Institute of Atmospheric Physics of the Czech Academy of Sciences, Prague, Czech Republic

4 LESIA, Observatoire de Paris, Université PSL, CNRS, Sorbonne Université, Université de Paris, Meudon, France

${ }^{5}$ LPC2E, CNRS, University of Orléans, 3A avenue de la recherche scientifique, Orléans, France

${ }^{6}$ Radboud Radio Lab, Department of Astrophysics, Radboud University, Nijmegen, The Netherlands

7 Johns Hopkins Applied Physics Lab, Laurel, MD 20723, USA

8 Space Research Institute, Austrian Academy of Sciences, Graz, Austria

9 Space Sciences Laboratory, University of California, Berkeley, CA, USA

10 Istituto per la Scienza e Tecnologia dei Plasmi (ISTP), Consiglio Nazionale delle Ricerche, Via Amendola 122/D, 70126 Bari, Italy

11 Space and Plasma Physics, KTH Royal Institute of Technology, 10405 Stockholm, Sweden
12 Faculty of Mathematics and Physics, Charles University, Prague, Czech Republic

13 Department of Astrophysics, Astronomy and Mechanics, Faculty of Physics, School of Science National and Kapodistrian University of Athens, 15783 Zographos, Greece

14 Department of Climate and Space Sciences and Engineering, University of Michigan, Ann Arbor, MI, USA

15 National Observatory of Athens, IAASARS, Metaxa and Vas. Pavlou str., Pedeli, 15236 Athens, Greece

16 School of Physics and Astronomy, University of Glasgow, G12 8QQ Glasgow, UK

17 Department of Physics and Astronomy, University of Iowa, Iowa City, IA 52242-1479, USA

18 Physics Department, University of California, Berkeley, CA, USA

19 Department of Physics, Imperial College, SW7 2AZ London, UK

${ }^{20}$ Lunar and Planetary Laboratory, University of Arizona, Tucson, AZ 85721, USA

21 Department of Space and Plasma Physics, School of Electrical Engineering and Computer, Stockholm, Sweden

22 CNES, 18 Avenue Edouard Belin, 31400 Toulouse, France

23 Technische Universität Dresden, Wärzburger Str. 35, 01187 Dresden, Germany

24 Astronomical Institute of the Czech Academy of Sciences, Prague, Czech Republic

25 Institute of Atmospheric Physics of the Czech Academy of Sciences, Prague, Czech Republic

26 Jet Propulsion Laboratory, California Institute of Technology, Pasadena, CA 91109, USA

27 European Space Agency (ESA), European Space Astronomy Centre (ESAC), Camino Bajo del Castillo s/n, 28692 Villanueva de la Cañada, Madrid, Spain 